Apidologie, 1979, 10 (1), 29-34.

\title{
INFLUENCE DE L'ISOLEMENT \\ SUR LA CONSOMMATION ALIMENTAIRE DES OUVRIÈRES D'ABEILLE
}

Der Einfluss der Isolierung auf die Futteraufnahme von Arbeitsbienen

\author{
Gérard ARNOLD \\ Université René Descartes \\ Laboratoire de Sociologie animale \\ Château d'Ivoy, Ivoy-le-Pré \\ 18380 La Chapelle d'Angillon
}

\begin{abstract}
RÉSUMÉ
L'absence des congénères provoque une diminution de moitié de la prise de nourriture des abeilles. Le groupement est donc un facteur stimulant la consommation alimentaire.

Cependant, il ne semble pas que la mortalité précoce des abeilles isolées soit directement due à un manque de nourriture, puisque certaines abeilles isolées vivent malgré une prise alimentaire réduite.
\end{abstract}

\section{SUMMARY}

THE EFFECT OF ISOLATION ON FOOD CONSUMPTION OF WORKER HONEY BEES

The absence of congeners provokes, among bees, a reduction of food intake by one-half. Grouping bees thus is a factor which stimulates feeding.

However it does not appear that a premature death is directly related to a lack of food, for certain bees survive despite a reduction of food.

\section{INTRODUCTION}

Il a été précédemment montré que les abeilles isolées depuis la naissance mouraient en général plus rapidement que les abeilles groupées par dix (ARNOLD, 1978). Par ailleurs, on a vu que les abeilles groupées avaient un poids supérieur à celui des abeilles 
isolées (ARNOLD, en préparation). Il était donc intéressant de savoir si les abeilles isolées consommaient la même quantité de nourriture que les groupées ou non, et si cela influençait leur durée de vie.

Dans nos expériences, la seule nourriture donnée aux abeilles était du candi, c'està-dire un aliment uniquement glucidique composé d'un mélange de sucre glace (3/4) et de miel (1/4). Le candi est d'ailleurs la nourriture employée par la plupart des auteurs ayant travaillé sur l'alimentation des ouvrières : MAURIzıO (1945, 1946), SitBon (1967), Roger (1971). De Groot (1953) utilise une pâte de saccharose contenant $20 \%$ d'eau. Dans certains cas, du pollen était ajouté au sucre dans des proportions variables.

Cependant, la plupart des auteurs ne donnant qu'une alimentation glucidique sans protéines, nous utiliserons la même méthode, de manière à pouvoir établir des comparaisons.

PAIN (1961, 1963), puis Roger (1971) ont fait des mises au point bibliographiques très complètes sur l'alimentation des ouvrières. Nous nous limiterons dans ces rappels aux travaux portant sur les abeilles encagées depuis la naissance, ce qui est le cas dans nos expériences.

Maurizio (1945, 1946), puis De Groot (1953) pensent que les jeunes abeilles consomment moins de nourriture que les abeilles âgées. Pour Maurizıo, les jeunes abeilles d'été ou d'hiver consomment environ $15 \mathrm{mg}$ de candi par jour, tandis que les vieilles consomment de 20 à $26 \mathrm{mg}$ de candi. Pour DE Groot, la consommation n'est que de 12,5 mg de saccharose pour les jeunes abeilles et, en moyenne, $32 \mathrm{mg}$ pour les vieilles abeilles. Par contre, WAHL (1963) affirme que la consommation est plus forte pendant la première semaine puis diminue par la suite.

ROGER (1971) indique qu'une abeille vivant dans un groupe de cinquante individus consomme en moyenne $14,6 \mathrm{mg}$ de candi par jour; elle consomme en outre $6,7 \mathrm{mg}$ par jour de pollen.

Srrbon (1967) avait comparé la consommation alimentaire d'abeilles isolées et d'abeilles groupées, mais il avait travaillé sur des abeilles hivernantes âgées de plusieurs mois, ce qui est très différent des abeilles mises en expérience à leur naissance. Il a montré qu'au bout de quinze jours d'expérience, les abeilles isolées ont consommé autant que les abeilles groupées par cinquante. SiтBON en conclut donc que la différence de mortalité observée entre les deux lots d'abeilles n'est pas due à une alimentation différente.

Il n'existe donc aucun travail portant sur la consommation de nourriture des jeunes abeilles isolées depuis la naissance.

\section{MATÉRIEL ET MÉTHODES}

\section{Matériel d'élevage :}

Les abeilles utilisées sont des abeilles d'été de race italienne: Apis mellifica ligustica Sp. Elles proviennent du rucher du Laboratoire de Sociologie Animale situé à Mittainville dans le département des Yve- 
lines (région parisienne). Les cadres de couvain sont transportés à l'étuve à $33^{\circ} \mathrm{C}$ où les abeilles éclosent. Les expériences se sont déroulées pendant l'été 1978.

Les abeilles isolées sont disposées dans des anneaux de verre de quatre centimètres de diamètre sur quatre centimètres de hauteur. Ceux-ci sont tapissés de papier filtre ainsi que le fond et fermés d'un couvercle en toile métallique que traverse un abreuvoir contenant de l'eau. Les abeilles groupées sont placées dans des tubes de quatre centimètres de diamètre sur huit centimètres de haut également munis de papier filtre et d'un couvercle traversé par un abreuvoir. Les abeilles y sont groupées par dix. L'ensemble des abeilles est maintenu à l'obscurité dans une étuve à $32^{\circ} \mathrm{C}$.

\section{Nourriture :}

Le candi est donné sous forme de boulettes pesant environ quatre cents milligrammes. Celles-ci sont déposées sur une coupelle en matière plastique constituant le fond de la cage des abeilles. L'ensemble coupelle plus boulette de nourriture est pesé d'abord au début de l'expérience, puis le cinquième jour, et enfin le dixième jour. Des boulettes témoins sont également déposées dans l'étuve afin de mesurer la perte de poids par évaporation de l'eau contenue dans le candi.

La consommation réelle des abeilles est donc déduite en soustrayant d'une part le poids de la coupelle, et d'autre part le poids de l'eau évaporée. Il est évident que pour les abeilles groupées, le poids de nourriture consommée qui est mesuré est celui de dix abeilles. Il faudra donc estimer la consommation moyenne d'un individu en divisant par dix.

\section{RÉSULTATS}

\section{Influence comparée du groupement et de l'isolement :}

Seize lots de dix abeilles ont été constitués, soit quatre-vingts abeilles isolées et quatre-vingts abeilles groupées par dix. Les résultats sont exprimés dans le tableau I où chaque nombre représente la quantité moyenne de nourriture consommée par individu et par jour.

Remarque : Aucune abeille groupée n'est morte pendant dix jours; par contre vingt abeilles isolées sont mortes (du troisième au cinquième jour).

Pour exprimer la consommation des isolées, on a donc divisé la consommation totale de chaque abeille morte par le nombre de jours où elle a vécu. Les résultats sont intégrés à ceux des abeilles restées vivantes dont la consommation moyenne est bien entendu basée sur cinq jours.

\section{Analyse statistique :}

Les consommations des abeilles isolées et celles des abeilles groupées ont été comparées à l'aide du test " $t$ » de STUDENT. La différence est très hautement significative pour chaque période :

$$
\begin{aligned}
& \text { du } 1^{\mathrm{er}} \text { au } 5^{\mathrm{e}} \text { jour }: t=4,7 ; \text { d.d.l. }=14 ; p<1 \% \text { } \\
& \text { du } 6^{\mathrm{e}} \text { au } 10^{\mathrm{e}} \text { jour }: t=4,8 ; \text { d.d.1. }=14 ; p<1 \% \text { o }
\end{aligned}
$$


TABL. 1 - Consommation quotidienne moyenne de candi par les abeilles isolées et les abeilles groupées (mg/jour/abeille).

TAB. 1 - Mittlere tägliche Zuckerteigaufnahme von isolierten und von gruppierten Bienen ( $\mathrm{mg} / \mathrm{Tag} / \mathrm{Biene})$

\begin{tabular}{|c|c|c|c|c|}
\hline \multirow[b]{2}{*}{ Lots } & \multicolumn{2}{|c|}{$\begin{array}{l}\text { Abeilles isolées } \\
\text { Isolierte Bienen }\end{array}$} & \multicolumn{2}{|c|}{$\begin{array}{l}\text { Abeilles groupées par dix } \\
\text { Bienen in Gruppen zu zehn }\end{array}$} \\
\hline & $\begin{array}{l}\text { Période du } 1^{\text {er }} \\
\text { au } 5^{\mathrm{e}} \text { jour }\end{array}$ & $\begin{array}{l}\text { Période du } 6^{\mathrm{e}} \\
\text { au } 10^{\mathrm{e}} \text { jour }\end{array}$ & $\begin{array}{c}\text { Période du } 1^{\text {er }} \\
\text { au } 5^{\mathrm{e}} \text { jour }\end{array}$ & $\begin{array}{l}\text { Période du } 6^{\circ} \\
\text { au } 10^{e} \text { jour }\end{array}$ \\
\hline Versuch & $\begin{array}{l}\text { Periode vom } \\
\text { 1.-5. Tag }\end{array}$ & $\begin{array}{l}\text { Periode vom } \\
\text { 6.-10 Tag }\end{array}$ & $\begin{array}{l}\text { Periode vom } \\
\text { I.-5. Tag }\end{array}$ & $\begin{array}{l}\text { Periode vom } \\
6 .-10 \text { Tag }\end{array}$ \\
\hline 1 & 5,2 & 6,4 & 9,2 & 11,1 \\
\hline 2 & 7,4 & 7,2 & 11,8 & 16,4 \\
\hline 3 & 6,1 & 6,8 & 9,4 & 9,7 \\
\hline 4 & 9,6 & 7,4 & 12,8 & 11,2 \\
\hline 5 & 6,6 & 7,9 & 15,7 & 20,8 \\
\hline 6 & 6,2 & 7,1 & 12,0 & 17,9 \\
\hline 7 & 4,4 & 9,8 & 11,7 & 17,0 \\
\hline 8 & 7,1 & 7,5 & 19,3 & 12,2 \\
\hline Moyenne & 6,5 & 7,5 & 12,7 & 14,5 \\
\hline Mittel & & & & \\
\hline
\end{tabular}

Ces résultats sont très nets : les isolées consomment en moyenne la moitié de la quantité prise par les groupées. Ainsi, pendant les cinq premiers jours : $6,5 \mathrm{mg}$ par jour pour les isolées et $12,7 \mathrm{mg}$ par jour pour les groupées; pendant les cinq jours suivants : 7,5 mg par jour pour les isolées et $14,5 \mathrm{mg}$ par jour pour les groupées.

On constate également qu'il existe des variations importantes entre les lots : pour les isolées, pendant la première période, une abeille du lot 7 a consommé en moyenne 4,4 mg par jour, tandis qu'une abeille du lot 4 a consommé $9,6 \mathrm{mg}$ par jour, soit plus du double. Pour les groupées, cette dispersion entre les lots existe également : ainsi, pour le lot 1, la consommation moyenne est de $9,2 \mathrm{mg}$ par jour, tandis que pour le lot 8 elle est de 19,3 mg par jour. Ces chiffres suggèrent de grandes variations individuelles entre les abeilles, aussi bien chez les isolées que chez les groupées.

Par ailleurs, si on compare la prise de nourriture entre les deux périodes, on constate que les abeilles isolées comme les abeilles groupées consomment un peu plus pendant la seconde période que pendant la première.

\section{Relation entre la consommation des isolées et leur durée de vie :}

Puisque les isolées consomment moins de nourriture et qu'elles meurent plus rapidement que les groupées, on peut se demander si leur mort précoce ne serait pas due à un manque de nourriture. 
$\mathrm{Au}$ cours d'une expérience portant sur trente abeilles isolées, la nourriture a été pesée quotidiennement pendant les trois premiers jours; puis le quatrième et le cinquième jour, la mortalité a été relevée. Deux lots d'abeilles ont été ainsi constitués :

lot $A$ : quatorze abeilles qui sont mortes pendant les $4^{\mathrm{e}}$ et $5^{\mathrm{e}}$ jour,

lot $\mathrm{B}$ : dix-sept abeilles toujours vivantes le $5^{\mathrm{e}}$ jour.

TABL. 2. - Consommation de nourriture (candi) pendant les trois premiers jours (mg)

TAB. 2 - Futteraufnahme (Zuckerteig) während der ersten drei Lebenstage (mg)

\begin{tabular}{|c|c|c|}
\hline & $\begin{array}{l}\text { Mesures individuelles } \\
\text { Individuelles Gewicht }\end{array}$ & $\begin{array}{c}\text { Moyenne } \\
\text { Mittel }\end{array}$ \\
\hline $\begin{array}{l}\text { Abeilles mortes le } 4^{\mathrm{e}} \text { et le } 5^{\mathrm{e}} \text { jour } \\
\text { (14 individus) }\end{array}$ & \multirow{2}{*}{$\begin{array}{l}11,8-15,9-16,9-16,9-17-18-19,8-19,8-19,9-21,2-24,1- \\
24,2-24,8-29,1\end{array}$} & \multirow[t]{2}{*}{19,9} \\
\hline $\begin{array}{l}\text { Am 4. und 5. Tag abgestorbene Bienen } \\
\text { (14 Individuen) }\end{array}$ & & \\
\hline $\begin{array}{l}\text { Abeilles vivantes le } 5^{e} \text { jour } \\
\text { (16 individus) }\end{array}$ & \multirow[t]{2}{*}{$\begin{array}{l}11,2-11,4-14,3-15,3-15,8-16-16,4-17,2-18,7-20,7- \\
23,5-28,3-28,5-30,6-32,1-32,3\end{array}$} & \multirow[t]{2}{*}{20,7} \\
\hline $\begin{array}{l}\text { Am 5. Tag noch lebende Bienen } \\
\text { (16 Individuen) }\end{array}$ & & \\
\hline
\end{tabular}

Analyse statistique :

L'utilisation du test « $r$ » de Bravais - Pearson montre qu'il n'y a aucune corrélation entre la consommation de nourriture et la survie. $(r=0,166$; d.d.1. $=28)$.

Il semble donc que la survie après le $5^{\mathrm{e}}$ jour d'expérience ne soit pas influencée par la quantité de nourriture consommée les jours précédents puisque certaines abeilles qui ont peu mangé vont survivre alors que d'autres qui ont mangé davantage vont mourir.

\section{DISCUSSION ET CONCLUSION}

Il n'est guère possible de comparer mes résultats avec ceux de SitBon (1967) puisqu'il a utilisé des abeilles hivernantes d'âge indéterminé alors que j'ai utilisé des abeilles naissantes. La physiologie de ces insectes est très différente, et il n'est pas étonnant que les effets de l'isolement ne soient pas les mêmes.

Les résultats concernant la prise de nourriture des abeilles groupées sont en accord avec ceux trouvés lors de précédents travaux. La valeur moyenne de 13,6 mg par jour se situe entre le résultat de De Groot (1953) qui est de $12,5 \mathrm{mg}$ et celui de RoGER (1971) qui trouve $14,6 \mathrm{mg}$. 
La survie des abeilles isolées, au moins pendant les cinq premiers jours, semble être indépendante de la quantité de nourriture consommée. Il y a donc une ou plusieurs autres causes qui interviennent pour expliquer la mortalité. Il semble que le poids à la naissance soit un facteur important : les abeilles les plus légères mourant en moyenne plus rapidement que les lourdes (ARNOLD, à paraitre). Mais ce facteur ne suffit pas à expliquer une telle différence de durée de vie entre les groupées et les isolées. L'absence de contacts avec les congénères et en particulier l'absence des échanges trophallactiques reste l'hypothèse la plus vraisemblable pour expliquer la mortalité plus rapide des isolées.

Reçu pour publication en octobre 1978.

Eingegangen im Oktober 1978.

\section{ZUSAMMENFASSUNG}

Frühere Arbeiten haben gezeigt, dass Arbeiterinnen, die von ihrem Ausschlüpfen an isoliert gehalten werden, früher sterben als Bienen aus Gruppen von zehn, und dass sie ein geringeres Gewicht haben.

In dieser Untersuchung werden zwei Fragen behandelt: Nehmen die isolierten Bienen dieselbe Quantität an Nahrung auf als die gruppierten?

Beeinflusst die Menge der aufgenommenen Nahrung die Lebensdauer?

Die dargebotene Nahrung bestand ausschliesslich aus Zuckern (Zuckerteig).

Unsere Versuche an Sommerbienen, die von ihrer Geburt an isoliert waren, haben ergeben, dass diese Bienen im Mittel nur die Hälfte der Futtermenge aufnehmen wie die gruppierten (Tab. 1). Die Überlebensdauer wird von der aufgenommenen Futtermenge nicht beeinflusst, denn manche Bienen, die weniger gefressen hatten, überlebten, während andere mit grösserer Nahrungsaufnahme starben (Tab. 2).

\section{BIBLIOGRAPHIE}

ARNoLD G., 1978. - Les variations annuelles dans l'effet de groupe chez l'abeille et l'origine de la mort précoce des isolées. Insectes Soc. 25 (1) 39-51.

ARNOLD G., (sous presse). - The influence of isolation upon the weight and the mortality of honeybees. $J$. Apic. Res.

Groot De A.P., 1953. - Protein and amino acid requirements of the honeybee. Physiol. Comp. Oecol. 3 (2) $1-90$.

MAURIzio A., 1945. - Trachtkrankheiten der Bienen : I-Vergiftungen bei einseitiger Tracht von Rosskastanien. Beih. Schweiz. Bienenztg., I (8) 337-368.

MAURIzIo A., 1946. - Beobachtungen über die Lebensdauer und den Futterverbrauch gefangen gehaltener Bienen. Beih. Schweiz. Bienenztg., 2 (13), 1-44.

PAIN J., 1961. - Sur la phéromone des reines d'abeilles et ses effets physiologiques. Thèse Sci. Nat. Paris $\mathrm{n}^{\circ}$ 4526, publ. in : Ann. Abeille, 4, 73-158.

PAIN J., 1963. - L'alimentation de la jeune abeille. Ann. Nutrit. Aliment. 17 (1), 307-312.

Roger B., 1971. - L'influence de la reine d'abeille sur la prise de nourriture des ouvrières accompagnatrices. Apidologie, 2 (2) 123-155.

SitBon G., 1967. - L'effet de groupe chez l'abeille. Ann. A beille, 9 (1) 5-36.

WAHL O., 1963. - Vergleichende Untersuchungen über den Nährwert von Pollen, Hefe, Sojamehl und Trockenmilch für die Honigbiene. Bienenforsch. 6 (8) 209-280. 